\title{
Commento al caso clinico: Setting ad assetto variabile. La chat dei sogni in psicoterapia: il caso di Adriana
}

\author{
Gianfranco Bruschi*
}

L'incontro in terapia, come tutti gli incontri, è l'inizio di una nuova storia e una storia si descrive e si disvela nel tempo e nello spazio di una relazione, secondo una direzione che, contemporaneamente, ne esclude altre. È quello che l'autore del caso clinico presentato ci dice, raccontando come vede e sente la presenza e il manifestarsi della persona in terapia, con una sensibilità che rimanda all'estetico, nel senso della sensibilità al rapporto che si crea nell'incontro con l'altro. Tale descrizione non può che sollecitare in me un altro incontro, quello che costruisco nella mia mente, immaginando l'autore, persona-terapeuta e la persona-paziente, nel loro interagire, lasciando emergere così un insieme di sensazioni, immagini, emozioni e pensieri che dischiudono il mio incontro estetico con questa storia.

I particolari della descrizione riportata iniziano ad intrecciarsi nel presente dell'osservazione dell'autore: il corpo, il volto (confinato all'espressività degli occhi, della fronte, dei respiri, come oggi da qualche tempo siamo costretti a fare, nella prossimità della relazione di aiuto non mediata), le movenze ed i gesti, ma soprattutto ciò che non si vede e che siamo costretti ad immaginare, che il terapeuta stesso, autore dello scritto in questione, raccoglie come un significante fondamentale dello scambio relazionale tra madre e figlio, il sorriso. È quel sorridere, come succederà poi in ogni relazione, che a guisa di frattale riproduce in un'infinità di posizioni la stessa dinamica che da' luogo a diverse percezioni, che divengono forme nel nostro apprendere e quindi nella nostra descrizione. Forme quasi indistinguibili all'inizio, che si definiscono via via che il processo relazionale si disvela.

Così succede in terapia e anche in questo percorso psicoterapico, dove l'autore riporta il suo procedere per ipotesi sulla base delle osservazioni e

*Psicologo psicoterapeuta Azienda USL di Parma, docente di Psicologia Clinica Corso di Laurea in Infermieristica Università degli Studi di Parma, docente Scuola di Specializzazione in Psicoterapia sistemico relazionale IDIPSI di Parma.

E-mail: gianfranco.bruschi@gmail.com 
delle interazioni che a mano a mano si costruiscono nelle varie sedute. E mi ritrovo a pensare come tante volte mi sono sentito anch'io limitato dal non vedere il sorriso, sviluppando una sorta di nuova sensibilità allo sguardo e alle espressioni del corpo nel suo respirare bendato.

L'ipotesi del funzionamento psichico della paziente, così come descritta dall'autore, rimanda a quello che posso vedere della relazione terapeutapaziente, come lo stesso autore riconosce in una prospettiva relazionale ('io ti racconto i sogni ma questi racconti riguardano te e me'): ambedue si ritrovano nel gioco relazionale vissuto ed appreso dalla paziente, un gioco in cui lei oscilla tra l'esperienza 'fusionale' del rapporto col padre, 'eccitatoria' e creativa, e quella 'simbiotica' della relazione con la madre, in conflitto, dolorante e depressiva.

Il lavoro descritto parla infatti della costruzione di uno spazio e di un tempo relazionale dove il terapeuta cerca di vestire le parti paterne del desiderio e quelle materne del limite, aiutando la paziente a stare nel presente della relazione con i suoi limiti che possono essere accettati, e quindi attraversati, senza il dolore che il corpo di Adriana vive proprio in virtù dell'essere non vista, non sostenuta, anzi chiusa e senza forze nelle sue potenzialità.

Vista e sostenuta come si era sentita invece anche con il compagno, a quanto viene riportato. Poi però Adriana, dopo l'interruzione della relazione con il padre di sua figlia, è costretta a rientrare in casa sotto le ali protettrici materne, che però appaiono come le ali ruvide e fredde di una madre che pare non aver conosciuto affetto e calore. E anche Adriana si trova in questa condizione, non essendo ancora donna-madre, ma ragazza-madre, a replicare ancora una volta, come una sorta di mimesi socio-affettiva, la transazione tra una mamma che non voleva esserlo e una figlia che non è vista quindi dalla sua stessa madre.

La descrizione fatta dall'autore mi rimanda, a differenza di quello che sembra succedere con la madre della paziente, il tentativo di rispondere all'attivazione del sistema di accudimento attraverso il dolore fisico, con la costruzione di uno spazio complesso, dove si susseguono esperienze sul versante eccitatorio e creativo, che coesistono in dialogo con altre parti in cui si negoziano significati dolorosi di contatto ed accettazione della realtà.

Relazione terapeutica come spazio dove integrare l'area del desiderio con quella della realtà e dei suoi limiti. Nel gioco tra sogni e realtà del setting terapeutico, si ricostruisce così un contesto in cui Adriana ha la possibilità di sperimentare ambedue i versanti e cercare di realizzare desideri che siano realistici, senza inseguire una guarigione 'magica' della realizzazione del desiderato, ma accettando di vivere la delusione ed il dolore di una revisione della sua vita nel setting terapeutico.

Essere in relazione è caratteristico della nostra dimensione umana, come anche di altri 'soggetti ecologici' come dice Morin (2009), e può essere visto 
come il motore, e quindi transizione, di esperienze che viviamo e che rimangono scritte nelle nostre memorie attraverso lo sperimentare di emozioni. Così l'apprendere diventa forma di esperienza, che siamo capaci di replicare e riconoscere, talvolta, e con il procedere del vivere sempre di più anche di descrivere. L'apprendimento assume così una forma che diviene 'tecnica', nel senso di strategia utile, quindi dipendenza (nel senso umano e usuale del termine) da una pratica imparata, che diviene appartenenza ad un repertorio di azioni e relazioni conosciute. Il bisogno e il desiderio di realizzazione ed autonomia, non può essere indipendenza, a costo di negare la stessa appartenenza, che è il nostro essere attraverso la relazione. Fin dai primi respiri impariamo accostando analogicamente le esperienze e diamo loro una forma di senso che ci aiuta a ritrovare sicurezza come succede con un suono conosciuto. Così immagino il lavoro del terapeuta che viene riportato nello scritto in oggetto. Desiderio come forma di ideazione di qualcosa che non possiamo mai raggiungere se non attraverso un apprendimento della realtà con la quale siamo in relazione, che vuol dire anche distaccarsi da ciò a cui si è attaccati è da cui si è dipendenti. Staccarsi dal desiderio, quindi, per trovare la via percorribile attraverso la relazione da cui si dipende-a cui si appartiene, ma anche da cui ci si può separare per costruire nuove libere attribuzioni di senso e azioni, che si tendono e si ricombinano nella rete dei nostri significati emotivi.

Nella relazione terapeutica di cui parliamo, aggiungo anche me stesso e mi chiedo cosa connette me con il terapeuta e con la paziente e con essi anche tutto il loro mondo abitato di relazioni...

Sarebbe forse un lavoro infinito quello che mi aspetterebbe nel descrivere tutto questo insieme di connessioni, per cui devo darmi un limite. 'Il nostro corpo è la terra che abitiamo' mi è capitato di ascoltare in un qualche convegno di cui ora non ricordo il titolo. Quindi cerco di riflettere su quello che il territorio del mio corpo, inteso come sensazioni, emozioni, pensieri, mi rimanda e tento di costruire una mappa di quello che il racconto fatto dal terapeuta sul caso vissuto (nella sua relazione terapeutica con Adriana), può voler significare in rapporto con la mia esperienza di transazione con una parte desiderante ed una depressiva.

Io stesso mi trovo ad immaginare come poter dialogare con una parte di me che insegue l'ideale e il desiderio ed un'altra che fa i conti con le possibilità e con i limiti del reale. Cosa succederebbe se quella parte si potesse materializzare e le si potesse parlare, ricostruendo il senso di un desiderare che ritrovo in Adriana e nel terapeuta (il desiderio di creare ed esprimere sé stessi, quello di ascoltare e rivedere creativamente ciò che emerge in una maniera nuova e quindi liberante)? E se si potesse parlare con la parte limitante della realtà di dolore, che è stata vissuta e forse non compresa da Adriana, ma anche da sua madre, e anche dal terapeuta e da me? Possiamo immaginare di stare là dove non è affatto comodo stare, in 
coesistenza con il dolore reale vissuto e il desiderio ideale, ma anche con il desiderio realizzato, nel dolore e nella libertà di scelte che sono state fatte che erano le sole che in quel momento era possibile fare?

Con tali domande mi sovviene come una vertigine, quella sensazione di non controllo e di paura di non essere capaci di stare, davanti alla quale si vorrebbe fuggire, ma che può essere affrontata nel setting terapeutico, che con la sua variabilità permette, come nel caso presentato, un dialogo di intreccio tra esperienza e possibilità con le diverse parti del sé delle persone coinvolte.

\section{BIBLIOGRAFIA}

Manghi, S. (2009). Il soggetto ecologico di Edgar Morin. Trento: Edizioni Erickson.

Conflitto di interessi: l'autore dichiara che non vi sono potenziali conflitti di interessi.

Approvazione etica e consenso a partecipare: non necessario.

Ricevuto per la pubblicazione: 28 agosto 2021.

Accettato per la pubblicazione: 7 settembre 2021.

${ }^{\circ}$ Copyright: the Author(s), 2021

Licensee PAGEPress, Italy

Ricerca Psicoanalitica 2021; XXXII:590

doi:10.4081/rp.2021.590

This article is distributed under the terms of the Creative Commons Attribution Noncommercial License (by-nc 4.0) which permits any noncommercial use, distribution, and reproduction in any medium, provided the original author(s) and source are credited. 